\title{
Atuação da Liga Acadêmica de Anatomia Clínica e Cirúrgica na pandemia: um relato de experiência
}

The acting of the Academic League of Clinical and Surgical Anatomy in the pandemic: an experience report

Actuación de la Liga Académica de Anatomía Clínica y Quirúrgica en la pandemia: un informe de experiencia

\section{Resumo}

Devido à Pandemia Covid-19, no Brasil, as atividades presenciais dos cursos de medicina e ligas acadêmicas foram suspensas, ambas enfrentaram dificuldades para readaptar suas atividades ao formato remoto/virtual. O presente artigo objetiva relatar a experiência de atuação da Liga de Anatomia Clínica e Cirúrgica da Universidade Federal do Paraná, Campus Toledo, com ênfase na adaptação das atividades presenciais ao ambiente remoto/virtual de ensino e nas contribuições ao aprendizado da anatomia, na pandemia. Trata-se de um estudo descritivo, transversal, retrospectivo de caráter qualitativo, um relato de experiência. Realizou-se, na pandemia, inúmeras atividades em plataformas online, aulas quinzenais, majoritariamente abertas ao público e certificadas, postagens de materiais revisionais no Instagram da liga, como "AnatoCards" e divulgação de aulas/eventos. Realizou-se, de modo on-line, a Primeira Jornada de Anatomia Clínica e Cirúrgica do Oeste do Paraná, com palestrantes de diferentes especialidades médicas e regiões do país, e alcance superior a 2500 pessoas. Criação do “Anatodúvidas", em 2021, no Instagram, projeto de extensão da liga, que democratizou o acesso ao conhecimento anatômico, embasado cientificamente, em linguagem acessível, à comunidade. Efetuou-se uma matéria em Jornal da Região para ampliar a visibilidade da liga para milhares de pessoas. O incentivo à pesquisa resultou em duas publicações de resumos em Congressos de Medicina. Portanto, a atuação da liga na pandemia contribuiu ao amparo dos pilares ensino, pesquisa e extensão, e estimulou o engajamento dos discentes. Isso 
resultou em ampla promoção, extensão e aprimoramento dos conhecimentos anatômicos, contribuindo na formação médica mais humana e completa, beneficiando amplamente à comunidade.

Palavras-chave: COVID-19; SARS-CoV-2; Anatomia humana; Liga acadêmica; Mídias digitais; Ensino.

\begin{abstract}
Due to the Covid-19 Pandemic, in Brazil, the face-to-face activities of medical courses and academic leagues were suspended, both faced difficulties to readjust their activities to the remote/virtual format. This article aims to report the experience of the Clinical and Surgical Anatomy League at the Federal University of Paraná, Toledo Campus, with an emphasis on adapting face-to-face activities to the remote/virtual teaching environment and on contributions to the learning of anatomy in the pandemic. This is a descriptive, cross-sectional, retrospective, qualitative study, an experience report. During the pandemic, numerous activities were carried out on online platforms, biweekly classes, mostly open to the public and certified, posts of revisional materials on the league's Instagram, such as "AnatoCards" and dissemination of classes/events. The First Conference on Clinical and Surgical Anatomy of the West of Paraná was held online, with speakers from different medical specialties and regions of the country, reaching more than 2500 people. Creation of "Anatodúvidas", in 2021, on Instagram, an extension project of the league, which democratized access to anatomical knowledge, scientifically based, in accessible language, to the community. A story was published in Journal Local to increase the league's visibility to thousands of people. The research incentive resulted in two publications of abstracts in the Congress of Medicine. Therefore, the league's role in the pandemic contributed to the support of the teaching, research and extension pillars, and stimulated the engagement of students. This resulted in a wide promotion, extension, and improvement of anatomical knowledge, contributing to a more humane and complete medical education, broadly benefiting the community.
\end{abstract}

Keywords: COVID-19; SARS-CoV-2; Human anatomy; Academic League; Digital media; Teaching.

\begin{abstract}
Resumen
Debido a la Pandemia del Covid-19, en Brasil, se suspendieron las actividades presenciales de los cursos de medicina y las ligas académicas, ambos enfrentaron dificultades para readaptar sus actividades al formato remoto / virtual. El presente artículo tiene como objetivo reportar la experiencia de la Liga de Anatomía Clínica y Quirúrgica de la Universidad Federal del Paraná, Campus de Toledo, con énfasis en la adaptación de las actividades presenciales al entorno de enseñanza remota / virtual y en las contribuciones al aprendizaje de anatomía en la pandemia. Se trata de un estudio descriptivo, transversal, retrospectivo y cualitativo, un informe de experiencia. Se realizó, en la pandemia, numerosas actividades en plataformas online, clases quincenales, mayoritariamente abiertas al público y certificadas, publicaciones de materiales de revisión en el Instagram de la liga, como "AnatoCards” y difusión de clases/eventos. Se realizó en línea el Primer Congreso de Anatomía Clínica y Quirúrgica del Occidente de Paraná, con ponentes de diferentes especialidades médicas y regiones del país, llegando a más de 2500 personas. Creación, en 2021, del "Anatodúvidas" en Instagram, un proyecto de extensión de la liga, que democratizó el acceso al conocimiento anatómico, con base científica, en lenguaje accesible, a la comunidad. Se publico una materia en el Períodico de la Región para ampliar la visibilidad de la liga ante miles de personas. El incentivo a la investigación se traddujo en dos publicaciones de resúmenes en Congresos de Medicina. Por lo tanto, la actuación de la liga en la pandemia contribuyó al apoyo de los pilares de la enseñanza, la investigación y la extensión, y estimuló la participación de los ligandos. Esto resultó en una amplia promoción, extensión y mejora del conocimiento anatómico, contribuyendo a una educación médica más humana y completa, beneficiando ampliamente a la comunidad.
\end{abstract}

Palabras clave: COVID-19; SARS-CoV-2; Anatomía humana; Liga académica; Medios digitales; Enseñanza.

\title{
1. Introdução
}

A doença da Covid-19 iniciou-se na cidade de Wuhan, província de Hubei, na China, em dezembro de 2019. Alastrouse no início de 2020 para vários países, passando a ser conhecida como a Pandemia da Covid-19, com impactos em esfera mundial. A doença é causada pelo novo Coronavírus-2 da Síndrome Respiratória Aguda Grave (SARS-CoV-2), que foi detectado pela China em janeiro de 2020 (OMS, 2020). A princípio, em janeiro de 2020, a Organização Mundial da Saúde (OMS) declarou o novo surto da Covid-19 como uma Emergência em Saúde Pública de Importância Internacional (ESPII), termo que foi usado pela sexta vez na história mundial e representa o nível mais elevado de alerta da OMS. É definido como um risco de saúde pública, que requer, potencialmente, uma resposta mundial coordenada, de acordo com as normas internacionais de regulamentação sanitárias (OPAS, 2020).

Porém, não foi possível conter a disseminação do vírus para os diversos países do mundo, passando a ser chamada, pela OMS, em 11 de março de 2020, de Pandemia da Covid-19 (OPAS, 2020), que se tornou um importante marco na história da humanidade, passando o mundo a ser dividido em pré e pós início da pandemia. Isso representa as importantes mudanças 
mundiais que foram necessárias para garantir a saúde mundial, como o uso de máscaras individuais, distanciamento social durante o pico da pandemia, e uso frequente de álcool 70\% (OPAS,2021), seja em gel, para higienização das mãos, ou mesmo álcool líquido, utilizado para limpeza geral de utensílios, bancadas/mesas e cadeiras.

A Liga Acadêmica de Anatomia Clínica e Cirúrgica (LAACC) da Universidade Federal do Paraná (UFPR), Campus Toledo, Paraná (PR), foi fundada em 13 setembro de 2018 com o intuito de aprofundar os conhecimentos em anatomia e correlacionar os conteúdos teóricos à prática médica, clínica e cirúrgica, possibilitando uma formação médica mais completa, visando o atendimento médico prestado de forma ampla e humana, centrado na pessoa e não na doença.

Desde 2018 até os dois primeiros meses de 2020, as atividades da liga eram realizadas presencialmente, contando com aulas quinzenais, ministradas por convidados ou pelos ligantes, bem como a execução de outras atividades, como práticas de sutura e dissecção anatômica, tanto em peças de animais, como em cadáveres humanos, estes pertencentes à uma universidade de uma liga parceira, localizada em Cascavel-PR. Ademais, em 2019, a LAACC desenvolveu o projeto de preparação e montagem de esqueletos humanos para o estudo em anatomia, o qual beneficiou tanto os membros da liga quanto os demais discentes da UFPR, Campus Toledo. Em 2020, com os reflexos da pandemia na modalidade de ensino, houve a necessidade de adaptar também as atividades e projetos da liga para o ambiente virtual, visando estimular a busca de aprendizados em anatomia humana e aplicar os conhecimentos teóricos adquiridos, de modo a minimizar as limitações do ensino remoto para o curso de medicina.

Esse artigo tem por objetivo relatar a experiência de atuação da LAACC, liga do curso de Medicina da UFPR, Campus Toledo, e suas contibuições ao aprendizado da anatomia, durante a pandemia da Covid-19, principalmente as atividades realizadas em 2021. Visando demonstrando como foi possível expandir o ensino em anatomia humana, por meio da utilização das mídias digitais para o ensino dessa disciplina de extrema importância para a formação médica.

\section{Metodologia}

Trata-se de um estudo descritivo, retrospectivo, transversal de caráter qualitativo, do tipo relato de experiência (Pereira et al., 2018). Sabendo que a pandemia resultou em profundas mudanças no processo de ensino-aprendizagem (Monteiro et al., 2021), as atividades da LAACC também foram modificadas: passaram a ser realizadas de forma virtual durante a pandemia. Para o planejamento e ajuste do cronograma, a diretoria da liga se reunia por meio de plataformas online, com frequência mensal ao longo do ano. As palestras foram realizadas por palestrantes convidados e pelos ligantes, sobre assuntos determinados previamente. As aulas ministradas pelos integrantes da liga consistiram em apresentação e discussão de relatos de caso, os quais abordavam temas como variação anatômica e malformações congênitas.

Para as reuniões de equipe e realização das palestras, utilizou-se essencialmente a plataforma digital Microsoft Teams, com acesso via e-mail institucional. O uso do Google Meet se restringiu à uma aula apenas, a pedido da palestrante convidada. As aulas eram gravadas e disponibilizadas no canal do Youtube da LAACC. A plataforma de transmissão ao vivo StreamYard, em associação com o YouTube, foi de grande utilidade para possibilitar a realização da I Jornada de Anatomia do Oeste do Paraná. De forma a facilitar o gerenciamento das inscrições dos participantes externos e encaminhamento de e-mails com lembretes e certificações, as aulas abertas eram criadas na plataforma virtual de eventos Even3.

$\mathrm{O}$ acesso dos ligantes às aulas realizadas pelo Microsoft Teams se dava pela própria equipe da LAACC criada na plataforma, onde todos os membros eram adicionados sem a necessidade de inscrição prévia. Já para as aulas com transmissão pelo YouTube, o link de aula era disponibilizado com antecedência no Instagram da LAACC e no grupo do WhatsApp com todos os membros da liga presentes. Para os participantes externos, a inscrição se efetuava pelo preenchimento de um formulário da plataforma Even3, cujo endereço de e-mail fornecido era posteriormente adicionado à equipe do Microsoft Teams ou para o qual era enviado o link de aula no YouTube. 
Durante todo o período de duração das palestras abertas ao público, era disponibilizado um formulário de dúvidas em que os espectadores poderiam expor questionamentos referentes ao tema da aula, de forma anônima. Ao final da palestra, as dúvidas eram repassadas ao palestrante para que pudessem ser sanadas. Além disso, a participação em aula e eventual certificação eram validados através do preenchimento do formulário de presenças, em que eram coletados os dados pessoais necessários para a geração e o envio do certificado de cada participante. O link do formulário era enviado pelo chat ao final da aula e ficava disponível para preenchimento por até trinta minutos após o encerramento da transmissão. Cada um dos certificados foi encaminhado individualmente aos participantes, por meio do e-mail da LAACC.

A página do Instagram da LAACC era administrada exclusivamente pelos membros da diretoria. Eram produzidas postagens frequentes, tanto para a divulgação das palestras e eventos da liga, como para a publicação de flashcards de anatomia, chamados de "AnatoCards", que consistem em cartões de perguntas e respostas curtas para a revisão de conteúdos. Para a elaboração de alguns flashcards, foram feitas parcerias com outras ligas da UFPR, Campus Toledo, com o intuito de incentivar a transdisciplinaridade e ampliar a difusão do saber anatômico entre os acadêmicos de medicina.

No início de 2021, foi criado o projeto de extensão “Anatodúvidas” vinculado à Liga, com a criação de uma página no Instagram própria. Através de interações com os usuários da rede e postagem de temas relacionados à anatomia, com enfoque em uma linguagem menos formal e mais acessível à comunidade, o projeto buscou solucionar as dúvidas do público, bem como estimular o engajamento dos indivíduos no seu processo saúde-doença. Os temas das publicações foram divididos e elaborados pelos membros da LAACC, de maneira que todos participaram de sua execução.

Em julho, foi iniciada a série de postagens “A Covid-19 e a Anatomia Humana”, com uma sequência de informações acerca dos impactos da doença nos mais variados sistemas do corpo. Mais recentemente, a série "Anatomia Aplicada às Especialidades Médicas" contou com a parceria de demais ligas acadêmicas da UFPR, Campus Toledo, com a participação dos respectivos ligantes na elaboração de temas específicos que permitissem o enfoque da endocrinologia, cardiologia, medicina da família e ginecologia e obstetrícia, por exemplo.

Em relação às publicações científicas, os membros da liga elaboram dois resumos para publicação em congressos científicos, os resumos se basearam em extensa pesquisa nas bases de dados Pubmed e Scielo. Em agosto de 2021, a Diretoria da LAACC, elaborou uma matéria sobre a LAACC e sua atuação para o Jornal do Oeste. Ademais, a diretoria da liga ficou responsável por elaborar um resumo escrito e um vídeo enviado à $12^{a}$ SIEPE - Semana Integrada de Ensino, Pesquisa e Extensão, da UFPR.

\section{Resultados}

\section{Ensino e extensão:}

Durante a pandemia da Covid-19, em 2020, a LAACC retomou suas atividades de modo virtual, mantendo o formato de aulas quinzenais, ministradas pelos ligantes e por profissionais convidados. Em 2020, destacaram-se as aulas por título “Anatomia do Sistema Nervoso Central e a Covid-19" e "Escrita Científica", ministradas por palestrantes externos convidados. Em 2021, devido à manutenção da pandemia da Covid-19, as atividades da liga mantiveram-se de forma virtual, com início em março do mesmo ano. Durante o primeiro semestre de 2021, todas as aulas foram ministradas por palestrantes da área médica, de diferentes regiões do Brasil. A maioria das palestras realizadas foram abertas à comunidade, proporcionaram e incentivaram a integração e a troca de conhecimentos com populações externas à liga e ao Campus Toledo, e também foram certificadas, contribuindo à formação do currículo acadêmico dos ouvintes.

Entre as palestras abertas, pode-se destacar as aulas "Aspectos Anatômicos na Neuroendocrinologia”, "A importância da Anatomia Clínica Aplicada na Pneumologia", “Anatomia relacionada à prática clínica na Reumatologia”, "Anatomia aplicada 
à Oftalmologia", "Cardiopatias Congênita e Anatomia", "Nefrologia e Anatomia”, "Cirurgia de Nervos Periféricos - Conceitos Básicos" e "Anatomia aplicada ao Trauma Ortopédico - Fraturas Expostas".

No segundo semestre do ano, as aulas foram mescladas, entre as ministradas pelos ligantes e por palestrantes convidados. Destacando-se as palestras abertas à comunidade, realizadas por convidados, "Anatomia Aplicada aos Cortes Seriados de Abdome", "Anatomia Aplicada à Urologia" e "Anatomia Aplicada às Emergências Obstétricas". As aulas ministradas pelos integrantes da liga permitiram o enriquecimento dos conhecimentos em anatomia a todos os integrantes da liga, e maior integração entre os membros da LAACC, aprimoramento da pesquisa e o desenvolvimento de novas habilidades, como oratória e ensino.

A transmissão da maior parte das aulas abertas à comunidade foi realizada pelo canal do YouTube da LAACC, proporcionou maior integração, adesão e satisfação do público participante, facilitou o acesso às palestras e reduziu os problemas de transmissão. As aulas permitiram a ampliação e o aprofundamento do conhecimento em anatomia e de suas aplicações às práticas na medicina, aos membros da LAACC e aos demais ouvintes das palestras.

A LAACC, em sua própria página no Instagram, durante a pandemia, utilizou as mídias digitais para fins educativos de forma considerável em comparação ao período pré-pandemia, contribuindo para a divulgação das atividades remotas da liga. Elaborou-se, por meio desta plataforma, a exposição e o convite às aulas e aos eventos organizados pela LAACC, a transmissão de informações para os ligantes e demais interessados e também a publicação de "AnatoCards", os quais consistem em postagens simples sobre um tema da anatomia, de forma a transmitir conhecimentos básicos da área de forma rápida e dinâmica.

Ademais, baseando-se no ensino e na extensão, no início de 2021, foi criado o projeto Anatodúvidas, proporcionando o acesso em anatomia à comunidade em geral. O projeto democratizou o acesso aos conhecimentos anatômicos, com embasamento científico, por meio de publicações realizadas, em sua página no Instagram, com linguagem menos formal, destinadas à comunidade. A troca de mensagens pela rede social do projeto com o público, permitiu a resolução direta das dúvidas do público solicitante, bem como contribuiu para o desenvolvimento de publicações acerca das dúvidas enviadas, tornando possível o alcance de um maior número de pessoas, acerca destes assuntos.

Além disso, na página do Instagram do Anatodúvidas, foram englobados inúmeros temas e solucionadas diversas dúvidas da comunidade em relação à anatomia. Ademais, houve a criação de três séries temáticas de postagens, “A Covid-19 e a Anatomia Humana", “A Anatomia das doenças neurodegenerativas" e "A Anatomia aplicada às especialidades médicas", realizada em parceria com outras ligas da UFPR Campus Toledo. Nessas séries, foram abordadas as principais patologias relacionadas às temáticas específicas e suas características, com destaque à anatomia, mas englobando também etiologia, fisiopatologia, sinais e sintomas, diagnóstico, prevenção e tratamento.

Somado a isso, entre as atividades de divulgação da liga durante a pandemia, houve a oportunidade de uma publicação no Jornal do Oeste do Paraná, acerca da LAACC e de suas atividades, como o projeto de extensão desenvolvido pela liga, que continua em vigência. Isto possibilitou que milhares de pessoas da comunidade pudessem conhecer a LAACC e suas atividades, e ampliou o conhecimento em anatomia humana à sociedade.

Durante a pandemia foi estabelecida a parceria com outras ligas acadêmicas da UFPR, Campus Toledo-PR, em que juntas realizaram postagens sobre anatomia aplicada às especialidades médicas. Estas postagens foram realizadas no perfil, no Instagram, da LAACC, por meio dos “AnatoCards” e no "Anatodúvidas”, por meio da série de publicações “A Anatomia aplicada às especialidades médicas- principais doenças". As parcerias permitiram a integração de diversos assuntos médicos, o aprofundamento e a aplicação dos conhecimentos anatômicos em cada uma das especialidades abordadas, possibilitou a união, o desenvolvimento interpessoal e a troca de conhecimentos entre os membros das ligas.

Além disso, a busca em promover a extensão do acesso aos conhecimentos em anatomia motivou o desenvolvimento da I Jornada de Anatomia Clínica e Cirúrgica do Oeste do Paraná (I JACCOP), um ciclo de palestras online que ocorreu entre os 
dias 13 e 15 de setembro de 2021, com transmissão via YouTube. O evento contou com sete palestrantes convidados de diferentes regiões do país e de diversas áreas de atuação médica, incluindo cirurgia plástica e torácica, médico legista, neurocirurgia, ortopedia, patologia e radiologia.

A I JACCOP aprofundou o conhecimento em anatomia humana aplicada às especialidades médicas, demonstrando a importância da disciplina na atuação profissional e integrou teoria à prática clínica e cirúrgica. O evento alcançou mais de 2500 pessoas, incluindo estudantes e profissionais da área da saúde, pertencentes às mais diversas universidades públicas e privadas, com abrangência até mesmo de outros países da América Latina, como Argentina, Bolívia e Paraguai. O evento teve um retorno positivo dos espectadores e contribuiu de forma significativa para a aquisição de conhecimentos e troca de experiências, além de desenvolver competências de gestão e trabalho em equipe, no que diz respeito à comissão organizadora da LAACC.

\section{Pesquisa:}

A LAACC contribuiu para o aprimoramento da pesquisa durante a pandemia da Covid-19, por meio da realização de dois resumos submetidos e aprovados em congressos. O resumo “A Covid-19 e os Principais Acometimentos Anatômicos no Sistema Nervoso Central e Periférico" foi publicado nos anais do Primeiro CONBRAMED - Congresso Online Brasileiro de Medicina, realizado entre 22 e 24 de março de 2021. E o resumo "A produção científica sobre os efeitos da Covid-19 no sistema nervoso central: revisão sistemática" foi aprovado para pôster digital no XIII Congresso Paulista de Neurologia, realizado entre os dias 27 e 29 de maio de 2021.

Os membros da diretoria da LAACC elaboraram um resumo escrito e um vídeo que foram enviados à $12^{\mathrm{a}} \mathrm{SIEPE}-$ Semana Integrada de Ensino, Pesquisa e Extensão, realizada de forma online entre os dias 22 a 26 de novembro de 2021 . O resumo foi aprovado para a $12^{\text {a }}$ SIEPE, onde a liga e suas atividades foram apresentadas pelos seus membros. Todos os membros da liga tiveram a oportunidade de participar deste importante evento para agregar conhecimento e integrar com indivíduos de outros cursos da UFPR.

Além disso, as publicações em anatomia realizadas pela LAACC em seu Instagram e na página “Anatodúvidas" da mesma mídia social, contribuíram para o incentivo constante à pesquisa em plataformas de pesquisa com elevado grau de confiabilidade, como Periódicos da Capes, Scopus, Elsevier, Pubmed, Scielo, UpToDaTe, Science Direct e Pubmed, resultando na ampliação do saber anatômico de todos os membros da LAACC.

\section{Discussão}

Com a mudança do calendário acadêmico para o meio virtual, devido à chegada do vírus SARS-CoV-2 no Brasil, em março de 2020, houve a necessidade da reinvenção da forma de atuação das ligas acadêmicas e do ensino médico como um todo. Dessa forma, houve uma reformulação das estratégias e práticas pedagógicas tradicionais, e o desenvolvimento de novas habilidades de ensino, adaptando-se às tecnologias da informação e comunicação (Santos et al., 2020).

A transição para um cronograma virtual/remoto representou um desafio para o curso de medicina, o qual contempla muitas atividades à campo e práticas em laboratório em sua grade curricular, com o intuito tanto de desenvolver habilidades técnicas quanto de aproximar o estudante do público para o qual prestará serviços em saúde. Com o intuito de ultrapassar limitações físicas e de oportunizar a utilização da tecnologia digital no âmbito de ensino, a LAACC, buscou remanejar-se para possibilitar a troca de conhecimentos interdisciplinares, interprofissionais e interinstitucionais, além de desenvolver ações educativas para a comunidade, beneficiando-se do alcance das redes sociais. Meneses et al. (2021), também remanejaram suas atividades de atuação durante a pandemia para ampliar a extensão e difusão do conhecimento em anatomia humana.

Consoante Bezerra et al. (2020), a utilização do ensino à distância no que se refere ao ensino de matérias de estudo prático, como a anatomia humana, exige mudança de hábitos e de comportamentos, porém, as alterações na tecnologia durante 
a pandemia permitiram que os indivíduos pudessem estabelecer relações, independentemente de suas localizações, tornando possível o desenvolvimento da educação e do ensino, apesar dos entraves.

Inicialmente, utilizando-se do Microsoft Teams, oferecido pela UFPR aos discentes, foram realizadas aulas abertas à comunidade externa com professores de diversos municípios e instituições de ensino. As aulas foram divulgadas através do Instagram da liga (@laacc.ufpr), ver Figura 1. No entanto, devido aos empecilhos encontrados na utilização dessa plataforma, como a não visualização do chat pelos alunos que não possuíam o e-mail UFPR, foi iniciado o uso do site StreamYard, uma plataforma Live streaming, a qual direcionava a aula diretamente para o YouTube, não causando problemas quanto ao chat ou ao número de participantes. Além disso, visando contribuir para o currículo dos participantes, as aulas abertas foram certificadas.

Figura 1 - Instagram da LAACC com os posts de convite às aulas abertas.
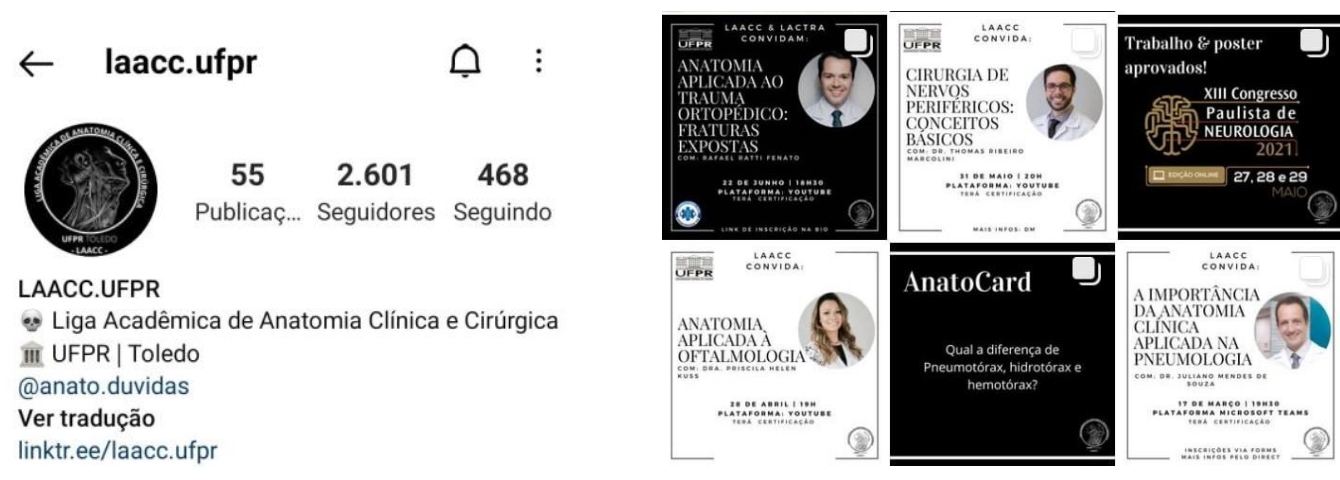

Fonte: Instagram da LAACC (2021).

Ademais, para a manutenção do projeto de extensão e sua continuidade e o tripé no qual se baseia (ensino, pesquisa e extensão), a liga procurou desenvolver o ensino por meio das aulas on-lines, a pesquisa com orientação do coordenador e a extensão, por meio de eventos que envolvessem a anatomia aplicada às especialidades médicas e o projeto Anatodúvidas.

Durante a graduação, a anatomia costuma ser apresentada de forma expositiva e pouco aplicada, levando a monotonia (Colares et al., 2019), dessa forma, buscou-se trazer a aplicação da anatomia nas especialidades, visto que é um recurso interessante para mostrar a importância da anatomia no dia a dia dos profissionais de saúde e torná-la cativante (Silva et al., 2018). A pesquisa é fundamental para desenvolvimento de uma liga, pois além da inserção no mundo científico, contribui para a criticidade do indivíduo que participa. Durante a pandemia, a produção científica mostrou-se fundamental para o estudo do vírus e controle da transmissão (Santos et al., 2019). Nesse contexto, a liga pode desenvolver a pesquisa, também voltada para as afecções da COVID-19 no corpo humano, por meio de duas revisões sistemáticas, um dos principais sistemas afetados durante a infecção pelo Novo Coronavírus e em quais países estava se realizando tal pesquisa, bem como outra voltada para analisar os danos causados no sistema nervoso central.

Ademais, a LAACC, assim como Meneses et al. (2021), utilizou as mídias digitais para fins educativos, de modo mais expressivo que o período pré-pandemia. Onde realizou publicações simples, rápidas e dinâmica sobre anatomia humana, conhecidas como "AnatoCards", e divulgou a atividades e eventos remotos para o público em geral, convidando-os a participarem das aulas/eventos realizados pela liga. Isso foi essencial para o crescimento da LAACC e sua atuação na comunidade, bem como para a difusão do conhecimento anatômico a milhares de pessoas das mais diferentes regiões do Brasil e para outros países.

A extensão mostra-se como uma oportunidade de alcance à comunidade, visto que nela é possível abordar o conhecimento científico de forma mais acessível, aproximando, leigos no assunto para a comunidade acadêmica (Cristofoletti \& Serafim, 2020). Assim, por meio de aulas abertas à comunidade em geral, seguindo o princípio de trazer a aplicação da anatomia 
na prática clínica e cirúrgica, também foi realizado o primeiro de evento de extensão intitulado "I Jornada de Anatomia Clínica e Cirúrgica do Oeste do Paraná - I JACCOP”, o qual trouxe profissionais de alguns estados brasileiros, apresentando a anatomia voltada às especialidades médicas, ver Figura 2.

Figura 2 - Palestra sobre "A anatomia aplicada à oncologia ortopédica" com o Dr. Rafael Ratti Fenato, durante a I Jornada de Anatomia Clínica e Cirúrgica do Oeste do Paraná (I JACCOP), transmitida no YouTube.

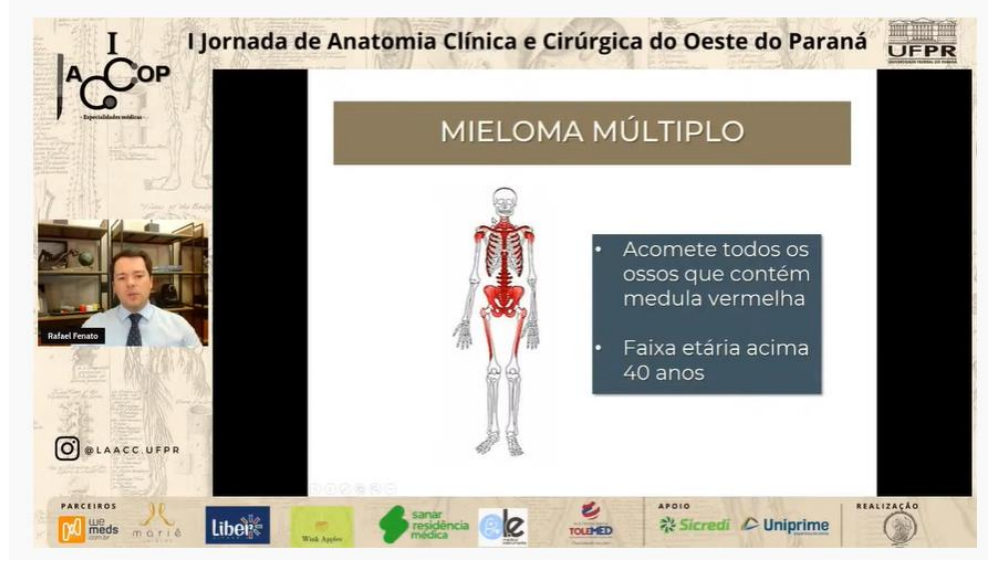

Fonte: YouTube da LAACC (2021)

O evento contou com mais de 2.500 inscritos de 25 estados brasileiros, Distrito Federal, Paraguai, Argentina e Bolívia. Além disso, a elaboração de um evento online com tais números foi de grande valia para o conhecimento aprimorado de gestão, elemento pouco estimulado durante os cursos de graduação, bem como trabalho em equipe e o desenvolvimento de habilidades pessoais (Hoffmann et al., 2021).

Além disso, sabendo que os processos comunicacionais estão sendo reinventados, de maneira a difundir conhecimento (Alves et al., 2018), foi idealizado o projeto Anatodúvidas (@anato.duvidas), um perfil no Instagram criado com o objetivo de sanar as dúvidas da população (leiga e estudantes) quanto ao estudo da anatomia, utilizando-se de uma linguagem simples e muitas imagens, ver Figura 3. A última série do Anatodúvidas, intitulada "A anatomia aplicada às especialidades médicas principais doenças", foi realizada em parceria com outras ligas da UFPR-Campus Toledo: a Liga Acadêmica de Ginecologia e Obstetrícia, a Liga Acadêmica de Medicina de Família e Comunidade, a Liga Acadêmica de Cardiologia e a Liga Acadêmica de Endócrinologia e Metabologia; fomentando o trabalho em equipe e a boa relação com outras ligas. 
Figura 3 - Perfil do Anatodúvidas no Instagram.
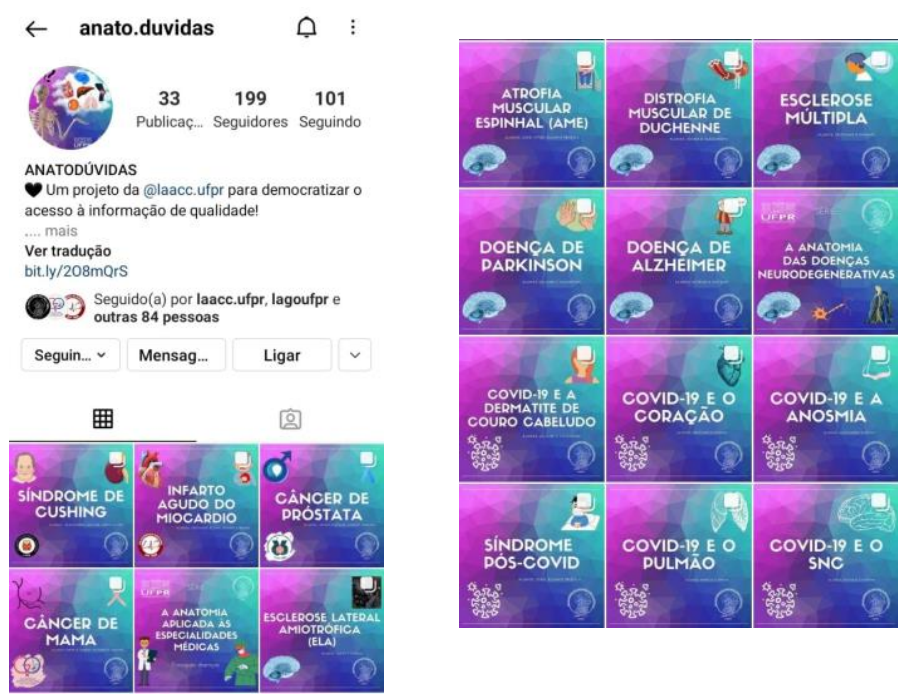

Fonte: Instagram do Anatodúvidas (2021).

Conforme Medeiros et al. (2020) relataram em seu relato de experiência de monitoria de anatomia humana durante a pandemia da Covid-19, os receios e preocupações em relação ao ensino remoto se dão não somente pela necessidade de inovar e de se adaptar às mudanças, mas também pela incerteza quanto à produtividade que as aulas e atividades tem para os alunos. Diante disso e das atividades da liga supracitadas, nota-se que, embora as medidas restritivas impedissem a realização de determinadas atividades, a liga conseguiu, de forma adaptada, dar continuidade ao tripé de ensino-pesquisa-extensão.

A divisão de tarefas, o engajamento dos membros e a formulação de novas ideias foram essenciais para a execução do cronograma, que inclui aulas abertas à comunidade externa e fechadas aos discentes da UFPR, além da escrita de artigos científicos, eventos de extensão e publicações informativas em redes sociais. Isso somente foi possível devido à sistematização do trabalho em equipe e o desenvolvimento de habilidades interpessoais, tanto para a discussão dos projetos quanto para o con vite aos palestrantes e intermediação durante a transmissão das aulas. As competências técnicas envolveram ainda o aprendizado no manejo das novas plataformas digitais, produção de conteúdo multimídia e controle sobre a organização dos ambientes virtuais, uma vez que, assim como apontado por Bottino (2021), foi imprescindível a utilização de tecnologias digitais de informação e comunicação no ensino remoto.

A variedade de temas explorados durante as aulas e ao longo da I JACCOP permitiram a inserção dos estudantes na disciplina de anatomia, a qual se evidenciou imprescindível independentemente da especialidade médica a ser escolhida. De acordo com Martelli et al. (2019), a anatomia humana é uma disciplina de fundamental importância para os inúmeros cursos dentro da área da saúde, consistindo em uma base comum de aprendizado, ao agregar conhecimento amplo sobre as estruturas que compõem o corpo humano.

Com o intuito de expandir o acesso à informação, os conteúdos elaborados pelos integrantes da LAACC e divulgados pela página Anatodúvidas, no Instagram, permitiram alcançar um público diversificado. Segundo Silva e Gouveia (2020), as redes sociais online são de fácil acesso à parte significativa da população, de simples manuseio e alcance geográfico das informações disponibilizadas. Ao considerar-se que as publicações divulgadas pelo Anatodúvidas possuíam embasamento na literatura científica (as buscas incluíram as bases de dados PubMed, Scopus, Science Direct, UpToDate e Scielo), esse projeto de extensão constituiu-se como importante estratégia no combate à desinformação e às fake news. Conforme Pulido et al. (2020), 
o impacto das fake news nas mídias sociais é uma questão de saúde pública, pois pode interferir na eficácia de programas, campanhas e iniciativas que visam à saúde, sensibilização e bem-estar dos cidadãos.

Dessa forma, a atuação da liga não se restringiu à comunidade acadêmica, mas teve o objetivo de propagar informação de qualidade acerca do funcionamento do corpo humano e sanar algumas dúvidas enviadas pelos próprios usuários das redes, relacionadas tanto à anatomia quanto à COVID-19. Portanto, foi possível associar o conteúdo da página com a educação em saúde e a demanda espontânea dos usuários. Isso também proporcionou a aproximação do ensino médico com a realidade da população, enfatizando os três pilares regidos pelo Sistema Único de Saúde (SUS), que são a integralidade, equidade e universalidade do cuidado.

No tocante ao intercâmbio de experiências, a realização de atividades por meio de plataformas online oportunizou a interação entre estudantes de medicina e palestrantes de diferentes universidades brasileiras, encurtando barreiras que até então possuíam limitações físicas. O contato entre instituições de ensino e as discussões entre profissionais especializados agregaram de forma significativa a formação teórica-prática dos membros da LAACC e demais acadêmicos envolvidos em suas atividades, complementando o conhecimento médico em seus aspectos técnicos, éticos, humanísticos e culturais.

Ademais, os membros da liga participaram do evento 12ª SIEPE - Semana Integrada de Ensino, Pesquisa e Extensão, que ocorre anualmente com a participação de toda UFPR, e cada um de seus Campus, e é promovido para divulgar as atividades e programas acadêmicos coordenados pelas diferentes Pró-Reitorias da universidade. Na edição de 2021, o evento ocorreu de forma online entre os dias 22 a 26 de novembro (UFPR, 2021), contando com a realização de mesas de conversa, compartilhamento de relatos de experiências e desenvolvimento de projetos acadêmicos e científicos durante a pandemia. A LAACC ficou responsável por elaborar um resumo em formato escrito e em vídeo, ambos enviados, aprovados e apresentados na $12^{\text {a }}$ SIEPE pelos membros da diretoria.

\section{Conclusão}

Diante do cenário pandêmico da COVID-19 e a implantação do ensino remoto, várias foram as adaptações necessárias no ambiente acadêmico, de forma a associar os aprendizados teóricos à prática médica. Com o intuito de prosseguir com o objetivo da liga de complementar o tripé ensino, pesquisa e extensão, a LAACC se propôs a reorganizar o seu cronograma de atividades e eventos, adotando como suporte as plataformas digitais.

Embora se apresente desafiador e complexo, o ensino da anatomia humana no contexto remoto possibilitou aos integrantes da equipe o desenvolvimento de habilidades técnicas e sociais, por meio da busca de novas alternativas de ensino. Permitiu ainda a aplicação do conhecimento anatômico às diversas áreas da medicina, de caráter clínico e cirúrgico, reiterando a importância da anatomia na formação profissional. Ademais, a grande abrangência das mídias digitais ampliou o acesso à informação de qualidade, de modo a beneficiar tanto a sociedade civil quanto a comunidade acadêmica.

Além disso, se oportunizou a intercambialidade de saberes e experiências entre estudantes e profissionais de diferentes universidades, além de aproximar o cuidado médico com as reais necessidades da população. A partir do que foi relatado, a atuação da LAACC durante a pandemia visou contribuir para a formação médica em seu aspecto holístico, contemplando a fundamentação teórica e também os pilares de integralidade, equidade e universalidade no atendimento médico prestado aos pacientes.

Por fim, a LAACC visa dar continuidade aos projetos elaborados e desenvolvidos durante a pandemia, como ao projeto de extensão da liga (o Anatodúvidas), à Jornada de Anatomia Clínica e Cirúrgica do Oeste do Paraná (JACOOP) e aos projetos de pesquisa. Somado a isso, as atividades presenciais da liga também terão seguimento, como a preparação e montagem de esqueletos humanos para estudo, as atividades de sutura e dissecção, entre outras. Afinal, essas ações poderão contribuir para o aprofundamento dos temas de anatomia humana em estudos futuros. 


\section{Referências}

Alves, L. A., Mota, F. M., \& Tavares, P. T. (2018). O Instagram como processo de engajamento das práticas educacionais: a dinâmica para a socialização do ensino-aprendizagem, Revista Científica da Fasete, 19.

Bezerra, A. D. da S., Oliveira, D. V. de, Carneiro, K. H. R., Lima, S. A., \& Moura, J. R. S. A. de. (2020). O ensino da anatomia humana em tempos de pandemia pelo vírus SARS-CoV-2. XXIX Encontro de Iniciação à Docência. Encontros Universitários da UFC, 5(4).

Bottino, C. F. dos S. (2021). Percepções sobre a educação em anatomia humana pós-pandemia do Covid-19: revisão sistemática da literatura. \#Tear: Revista De Educação, Ciência E Tecnologia, 10(1). https://doi.org/10.35819/tear.v10.n1.a4849

Colares, M. A. M., Mello, J. M. de, Vidotti, A. P., \& Sant'ana, D. de M. G. (2019). Metodologias de ensino de anatomia humana: estratégias para diminuir as dificuldades e proporcionar um melhor processo de ensino-aprendizagem. Arquivos Do Museu Dinâmico Interdisciplinar, 23(3), 140-160. https://doi.org/10.4025/arqmudi.v23i3.51527

Cristofoletti, E. C., \& Serafim, M. P. (2020). Dimensões Metodológicas e Analíticas da Extensão Universitária. Educação \& Realidade, 45(1). https://doi.org/10.1590/2175-623690670

Hoffmann, L. V., Ciorcero, N. M., Almeida, V. G., Zilles, A., Zem, F., \& Marques, C. M. (2021). Gestão e organização de evento acadêmico online no curso de medicina como ferramenta de atualização em tempos de pandemia: um relato de experiência. Brazilian Journal of Development, 7 (7), 65304-65312. https://doi.org/10.34117/bjdv7n7-007.

Martelli, A., Hunger, M. S., Delbim, L. R., Magalhães, R. A., \& Zavarize, S. F. (2019). Percepção dos acadêmicos dos cursos da área da saúde de uma Instituição de Ensino Superior acerca da Disciplina de Anatomia e sua influência na formação profissional. Archives of health investigation, 8(7). https://doi.org/10.21270/archi.v8i7.3270

Medeiros, J. H. B. de, Macedo, L. M., Chaves, N. C. B, Lima, B. R. N., Junior, M. F. de F., Oliveria, F. H. L. de, Rodrigues, A. Y. F., \& Lopes, C. M. U. (2020). Desafios do ensino superior durante a pandemia do covid-19: relato de experiência da monitoria de anatomia humana. Brazilian Journal of Development, 6 (10), 84008-84015.https://doi.org/10.34117/bjdv6n10-731.

Meneses, J. R.F., Rocha, H. F. P., Silveira, K. E. L. da, Sousa, A. V. D. de, Sena, A. V. A., Miranda, M. F. A. de, Beltrão, B. C. R., Gomes, F. V. M., Pinto, A. S. B., Andrade, A. R. O. de, Mendes Junior, F. das C. C., Pereira Júnior, J. L., Lima, L. F. B., Garcês, T. C. de C. S., \& Nóbrega Neto, A. de P. R. (2021) . Learning estrategy of Human Anatomy in basic Medical curriculum in a pandemic context: experience report using instagram. Research, Society and Development, 10(7), e42110716923, 2021. https://rsdjournal.org/index.php/rsd/article/view/16923.Doi: 10.33448/rsd-v10i7.16923

Monteiro, J. A., Dias, M. C., Athie, G. R., Mendes, J. L., \& Silva, J. T. (2021). Relato da situação dos modos de ensino em cursos de medicina no estado de Goiás durante a pandemia. Itinerarius Reflectionis: revista eletrônica de graduação e pós-graduação em educação, 17 (3), $34-50$.

Novel Coronavirus (2019-nCoV): Situation Report-1 (2020). https://www.who.int/docs/default-source/coronaviruse/situation-reports/20200121-sitrep-1-2019ncov.pdf?sfvrsn=20a99c10_4

Organização Pan-Americana da Saúde (2020). Histórico da pandemia de Covid-19. https://www.paho.org/pt/covid19/historico-da-pandemia-covid-19

Organização Pan-Americana da Saúde (2021). Folha informativa sobre COVID-19. https://www.paho.org/pt/covid19

Pereira, A. S., Shitsuka, D. M., Parreira, F. J., \& Shitsuka, R. (2018). Metodologia da pesquisa científica. UFSM. https://repositorio.ufsm.br/bitstream/handle/1/15824/Lic_Computacao_Metodologia-Pesquisa-Cientifica.pdf?sequence=1 .

Pulido C. M., Eugenio L. R., Sama G. R., \& Carballido B. V. (2020). A New Application of Social Impact in Social Media for Overcoming Fake News in Health. International Journal of Environmental Research and Public Health, 17(7): 2430. https://doi.org/10.3390/ijerph17072430

Santos, A. de O., Barros, F. P. C. de, \& Delduque, M. C. (2019). A pesquisa em saúde no Brasil: desafios a enfrentar. Saúde Em Debate, 43(5), 126-136. https://doi.org/10.1590/0103-11042019s511

Santos, B. M., Cordeiro, M. E. C., Schneider, I. J. C., \& Ceccon, R. F. (2020). Educação Médica durante a Pandemia da Covid-19: uma Revisão de Escopo Revista Brasileira de Educação Médica, 44 (01).

Silva, I., \& Gouveia, F. (2021). Engajamento informacional nas redes sociais: como calcular? AtoZ: novas práticas em informação e conhecimento, 10(1), 94 102. http://dx.doi.org/10.5380/atoz.v10i1.76633

Silva, J. H. da, Foureaux, G., Sá, M. A. de, Schetino, L. P. L., \& Guerra, L. B. (2018). O ensino-aprendizagem da anatomia humana: avaliação do desempenho dos alunos após a utilização de mapas conceituais como uma estratégia pedagógica. Ciência \& Educação, 24(1), 95-110. https://doi.org/10.1590/1516731320180010007

Universidade Federal do Paraná (2021). 12 ${ }^{a}$ SIEPE - Semana Integrada de Ensino, Pesquisa e Extensão. http://www.siepe.ufpr.br/2021/ 